\title{
Evaluación de diversos tamaños de sitio de muestreo en inventarios forestales
}

\author{
Oscar A. Aguirre Calderón ${ }^{1}$ \\ avier J iménez Pérez ${ }^{1}$ \\ Eduardo J . Treviño Garza ${ }^{1}$ \\ Blas Meraz Alemán²
}

\begin{abstract}
RESUMEN
En un rodal de Pinus Cooperi Blanco, en la región de El Salto, Durango, México se realizó una evaluación de diferentes tamaños de sitios circulares de muestreo para inventario forestal. En una superficie de 4.4 ha se llevó a cabo un inventario total y se establecieron 36 sitios de 0.1 ha. Empleando un simulador de sitios de dimensiones fijas para inventarios forestales, se probaron tamaños de sitio de 0.01 a 0.1 ha en intervalos de 0.01 ha. De los resultados obtenidos se determinó un tamaño de sitio óptimo de 0.06 ha en base a los parámetros estadísticos del área basal.
\end{abstract}

PALABRAS CLAVE:

Inventarios forestales, tamaño de sitio, optimización, Pinus cooperi Blanco.

\begin{abstract}
An evaluation of different sampling plot sizes was carried out on a Pinus cooperi Blanco stand in the region of $\mathrm{El}$ Salto, Durango, Mexico to determine optimal plot size for forest inventory. A complete census was carried out in a 4.4 ha area and 36 plots of 0.1 ha were established. Plot sizes of 0.01 to 0.1 ha, with intervals of 0.01 ha were tested using a fixed dimensions plot simulator. Based on the statistical parameters of the basal area, optimal plot size was 0.06 ha.
\end{abstract}

KEY WORDS:

Forest inventories, sampling plot size, optimization, Pinus cooperi Blanco

1 Facultad de Ciencias Forestales, U.A.N.L. Apdo. Postal 41. 67700 Linares, N. L. México.

2 Instituto Tecnológico Forestal No. 1. Apdo. Postal 2. 34950 El Salto, Dgo. México. Manuscrito recibido para su publicación el 8 de J ulio de 1996 


\section{INTRODUCCION}

En el marco de la elaboración de planes de manejo forestal, el inventario de los recursos forestales reviste capital importancia para la toma de decisiones. En las áreas dedicadas a la producción maderable, tal inventario comprende una descripción de los rodales que incluye, además de la condición general del ecosistema, información de carácter dasométrico y epidométrico (básicamente edad, altura, calidad o índice de sitio, grado de densidad, incremento y existencias), destacando en este contexto la determinación del área basal del rodal. Este parámetro permite derivar de manera rápida el volumen del rodal (Kramer y Akça, 1987), así como estimar la condición de densidad del mismo (Aguirre y J iménez, 1995).

El área basal de los rodales puede determinarse a través de un censo o inventario total, o estimarse mediante la realización de un muestreo. El primer procedimiento proporciona resultados precisos, aunque se emplea sólo en rodales de gran valor o para fines de investigación dados los altos costos que implica. Convencionalmente, para fines prácticos se prefiere la aplicación de diversos métodos de muestreo para el inventario de rodales, empleándose básicamente sitios de muestreo de dimensiones fijas, métodos basados en distancias y sitios de dimensiones variables (Zöhrer, 1980; De V ries, 1986; Schreuder et al., 1993). En éstos se inventarían sólo porciones de un rodal y los resultados se extrapolan a la superficie total del mismo. El muestreo es menos costoso en tiempo y dinero que el inventario total; su precisión es función de la homogeneidad del rodal y el número de sitios de muestreo establecidos en un área dada.
Dada la importancia del inventario forestal para el desarrollo de estrategias de manejo, éste debe realizarse atendiendo a niveles de precisión establecidos optimizando tiempo y costos. En este contexto, la determinación del tamaño óptimo de sitio y del número de unidades de muestreo necesarias para lograr un grado de precisión determinado, destacan como importantes tareas de investigación.

\section{OBJ ETIVO}

El presente trabajo tiene como objetivo evaluar diversos tamaños de sitio de muestreo a fin de contribuir al desarrollo de un método óptimo de inventario, empleando como parámetro dasométrico de referencia, el área basal del rodal objeto de estudio.

\section{METODOLOGIA}

\section{Area de estudio}

El área de estudio se ubicó en las cercanías de El Salto, Durango, México. Para este trabajo, se delimitó una superficie de 4.4 ha en un rodal constituído por Pinus cooperi Blanco, especie de gran importancia económica en la región.

Los parámetros dasométricos del rodal analizado se presentan en la Tabla 1.

\section{Obtención de los datos}

En el área seleccionada se realizó primeramente un inventario total del rodal, obteniéndose el diámetro normal de la totalidad de los árboles en milímetros, para la determinación del área basal real.Para la evaluación de los diferentes tamaños de sitio, se establecieron unidades de muestreo de forma circular en la superficie mencionada, con una distancia de $35 \mathrm{~m}$ entre los centros de los mismos. El tamaño de estos sitios fue de 
TABLA 1. Datos del rodal investigado

Table 1. Parameters of the stand under study

\begin{tabular}{||c|c|c|c|c|c|c||}
\hline Area & Edad & $\mathrm{N}_{\text {ha }}$ & $\mathrm{h}$ & $\mathrm{d}_{\mathrm{g}}$ & $\mathrm{G}_{\mathrm{ha}}$ & $\mathrm{V}_{\text {ha }}$ \\
\hline $4.4 \mathrm{ha}$ & 53 años & 243 & $15 \mathrm{~m}$ & $28.5 \mathrm{~cm}$ & $16.94 \mathrm{~m}^{2}$ & $113 \mathrm{~m}^{3}$ \\
\hline
\end{tabular}

$\mathrm{N}_{\text {ha }}=$ número de individuos por hectárea
$\mathrm{d}_{\mathrm{g}}=$ altura
$\mathrm{G}_{\text {ha }}=$ diámetro medio del área basal
$\mathrm{V}_{\text {ha }}=$ volumen medio por hectárea

0.1 ha (radio $=17.84 \mathrm{~m}$ ), dado que esta dimensión es de empleo común en México y para fines de procesamiento de los datos como se describe en el siguiente inciso.

Dentro de cada unidad de muestreo de 0.1 ha se determinó el diámetro normal en milímetros de cada uno de los árboles, así como su distancia al centro del sitio en centímetros. En total se obtuvo información en 36 sitios de muestreo.

\section{Procesamiento de los datos}

La información producto del inventario se procesó empleando un simulador de sitios de muestreo para computadora personal, desarrollado en el Instituto de Crecimiento y Manejo Forestal de la Universidad de Gotinga, Alemania. La simulación se realiza para sitios de dimensiones fijas de diversos tamaños con base en los diámetros normales de los árboles del sitio, así como su distancia al centro del mismo (Akça y Sangen-Emden, 1986). Con base en estos parámetros, el programa determina los árboles que serán incluidos en un sitio de un tamaño dado, calculando luego el área basal de la muestra y el valor correspondiente por hectárea. De los valores de área basal por hectárea de todos los sitios se derivan automáticamente la media aritmética, la desviación estándar y el coeficiente de variación para este parámetro.

\section{RESULTADOS}

\section{Resultados del inventario total}

Los resultados del inventario total del área basal en el rodal objeto de estudio se presentan en la Tabla 2. La distribución de los árboles del rodal en categorías diamétricas de $5 \mathrm{~cm}$ se presenta en la figura 1. En el rodal ocurren árboles con diámetros normales entre 5 y $65 \mathrm{~cm}$, predominando la categoría de $25 \mathrm{~cm}$.

Tabla 2. Resultados del inventario total Table 2. Results of the census

\begin{tabular}{||c||}
\hline \multicolumn{2}{|c|}{ Area basal } \\
\hline Total: $74.54 \mathrm{~m}^{2}$ \\
\hline Por ha: $16.94 \mathrm{~m}^{2}$ \\
\hline
\end{tabular}




\section{Resultados de la evaluación de tamaño de} sitio

En la Tabla 3 se presentan los parámetros estadísticos: área basal media $\left(\mathrm{G}_{\mathrm{ha}}\right)$, desviación estándar $\left(\mathrm{S}_{\mathrm{g}}\right)$, coeficiente de variación $\left(\mathrm{S}_{\mathrm{g}} \%\right)$, error estándar $\left(\mathrm{E}_{\mathrm{g}}\right)$ y error estándar porcentual $\left(E_{g} \%\right)$ en función del tamaño de los sitios. Como puede observarse, debido a la estructura del rodal investigado, se obtuvieron altos valores para los coeficientes de variación.

En la figura 2 se presenta el coeficiente de variación del área basal en función del tamaño de los sitios. En principio, se observa una rápida reducción de este parámetro a medida que incrementa la superficie del área de muestreo. A partir de un tamaño de sitio de 0.03 ha, tal reducción se atenúa claramente. La línea presenta entre 0.06 y 0.08 ha un curso casi paralelo a la abscisa, disminuyendo luego en menor medida. La dependencia entre los coeficientes de variación y las dimensiones del sitio, es por lo tanto cada vez menor conforme se incrementa la superficie de la misma, observándose que a partir del tamaño de sitio de $0.06 \mathrm{ha}$, el coeficiente de variación del área basal disminuye muy poco.

Del resultado anterior puede derivarse que en el rodal investigado los sitios de 0.06 ha son los que representan el tamaño óptimo para la realización del inventario.

El número de sitios necesario para la estimación del área basal con un error estándar de $\pm 5 \%$ ó $\pm 10 \%$ para sitios circulares de diferentes tamaños se presenta en la figura 3.

Tabla 3. Parámetros estadísticos para el rodal investigado

Table 3. Statistical parameters for the stand under study

\begin{tabular}{|c|c|c|c|c|c|}
\hline $\begin{array}{c}\text { Tamaño de } \\
\text { sitio (ha) }\end{array}$ & $\mathrm{G}_{\text {ha }}$ & $\mathrm{S}_{\mathrm{g}}$ & $\mathrm{S}_{\mathrm{g}} \%$ & $\mathrm{E}_{\mathrm{g}}$ & $\mathrm{E}_{\mathrm{g}} \%$ \\
\hline 0.01 & 19.65 & 15.21 & 77.4 & 2.54 & 12.90 \\
\hline 0.02 & 19.44 & 10.49 & 54.0 & 1.75 & 9.00 \\
\hline 0.03 & 18.85 & 6.63 & 35.2 & 1.10 & 5.87 \\
\hline 0.04 & 17.41 & 6.43 & 36.9 & 1.07 & 6.15 \\
\hline 0.05 & 17.04 & 5.79 & 34.0 & 0.96 & 5.67 \\
\hline 0.06 & 16.97 & 4.78 & 28.2 & 0.80 & 4.70 \\
\hline 0.07 & 16.83 & 4.51 & 26.8 & 0.75 & 4.47 \\
\hline 0.08 & 16.42 & 4.38 & 26.7 & 0.73 & 4.45 \\
\hline 0.09 & 16.12 & 3.89 & 24.1 & 0.65 & 4.01 \\
\hline 0.10 & 16.02 & 3.48 & 21.7 & 0.58 & 3.62 \\
\hline
\end{tabular}

$\begin{array}{ll}\mathrm{G}_{\text {ha }} & =\text { área basal media por hectárea } \\ \mathrm{S}_{\mathrm{g}} & =\text { desviación estándar } \\ \mathrm{S}_{\mathrm{g}} \% & =\text { coeficiente de variación } \\ \mathrm{E}_{\mathrm{g}} & =\text { error estándar } \\ \mathrm{E}_{\mathrm{g}} \% & =\text { error estándar porcentual }\end{array}$




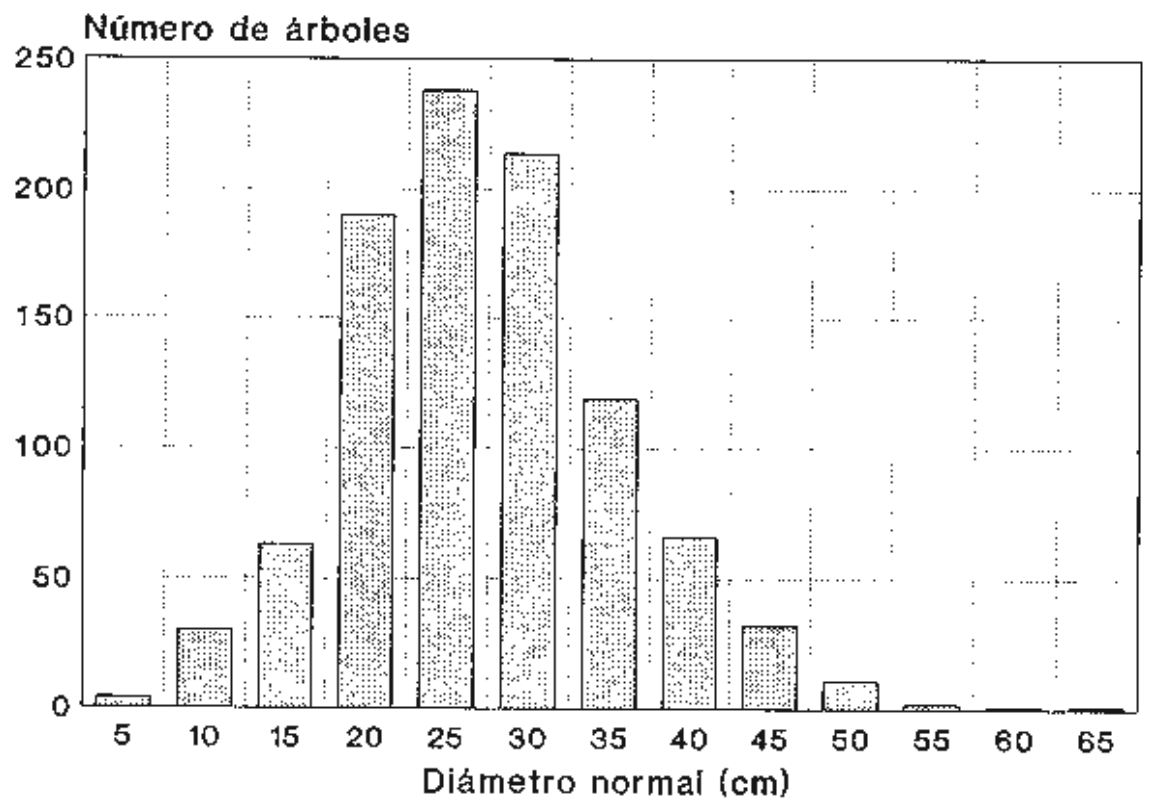

Figura 1. Distribución diamétrica del rodal (Diameter distribution of the stand)

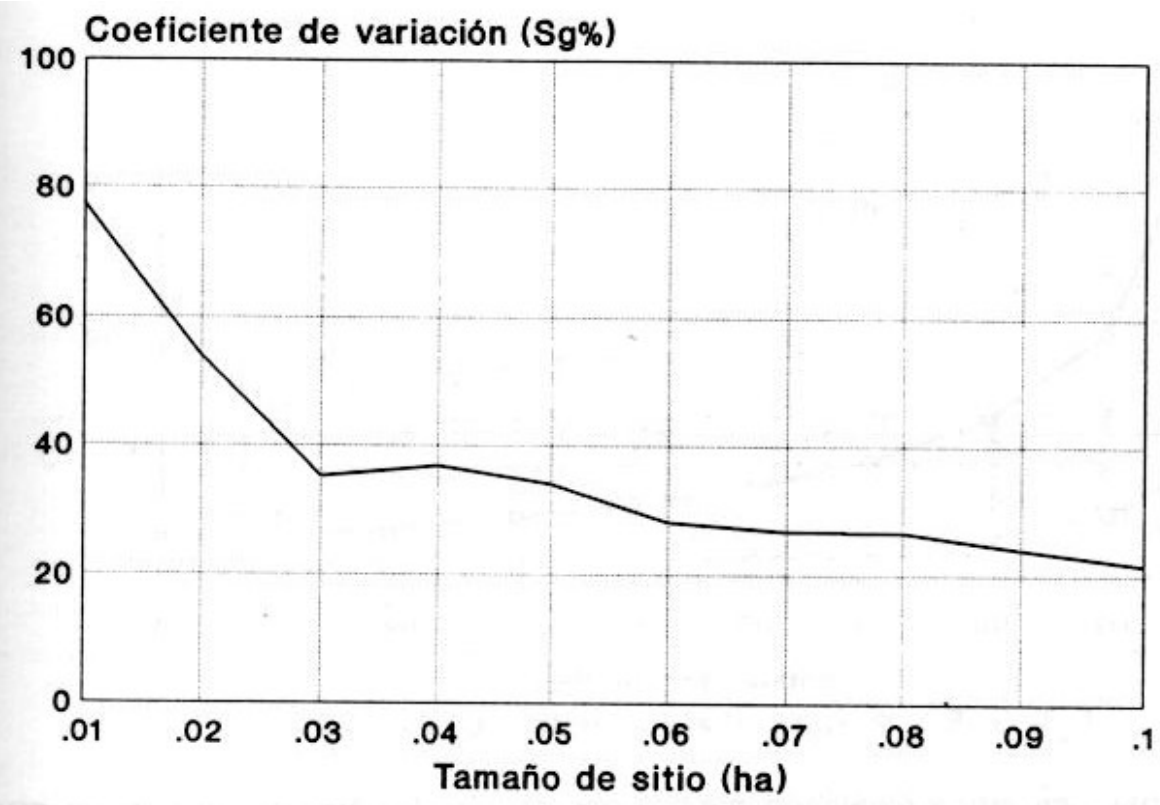

Figura 2. Coeficiente de variación del área basal media $\left(\mathrm{S}_{\mathrm{g}} \%\right)$ en función del tamaño de sitio (Variation coefficient of the basal area $\left(\mathrm{S}_{\mathrm{g}} \%\right)$ as a function of plot size) 
En ésta se observa que a medida que el tamaño de los sitios se incrementa, el número de sitios necesario para lograr un nivel de precisión dado, varía cada vez menos. Por otra parte, las cifras correspondientes a un error de $\pm 5 \%$ son sensiblemente superiores a los correspondientes a un error de $\pm 10 \%$, principalmente para sitios entre $0.01 \mathrm{y}$ 0.04 ha.

La figura 4 representa en forma esquemática el número de árboles promedio para los diferentes tamaños de parcela investigados. Con base en los resultados de estudios de tiempo realizados en investigaciones similares (O'Regan y Arvanitis, 1966; Zeide, 1980), el tamaño de sitio óptimo debe contener entre 10 y 25 árboles. El sitio 0.06 ha, que fue seleccionado de acuerdo con los valores del coeficiente de variación del área basal (Fig. 3), contiene en promedio 16 árboles de acuerdo a la curva de la figura 5, esto es, corresponde al tamaño de sitio óptimo.

La figura 5 muestra los intervalos de confianza (t. $2.0 "=0.05$ ) del área basal para diversos tamaños de sitio, así como el área basal promedio del inventario total (:).

El valor de área basal obtenido del inventario total se localiza en la mayoría de los tamaños de sitio en el rango del error estándar. Por otra parte destaca que tanto el error medio como los intervalos de confianza, apenas varían para los sitios entre 0.06 y 0.08 ha (ver Tabla 3 ).

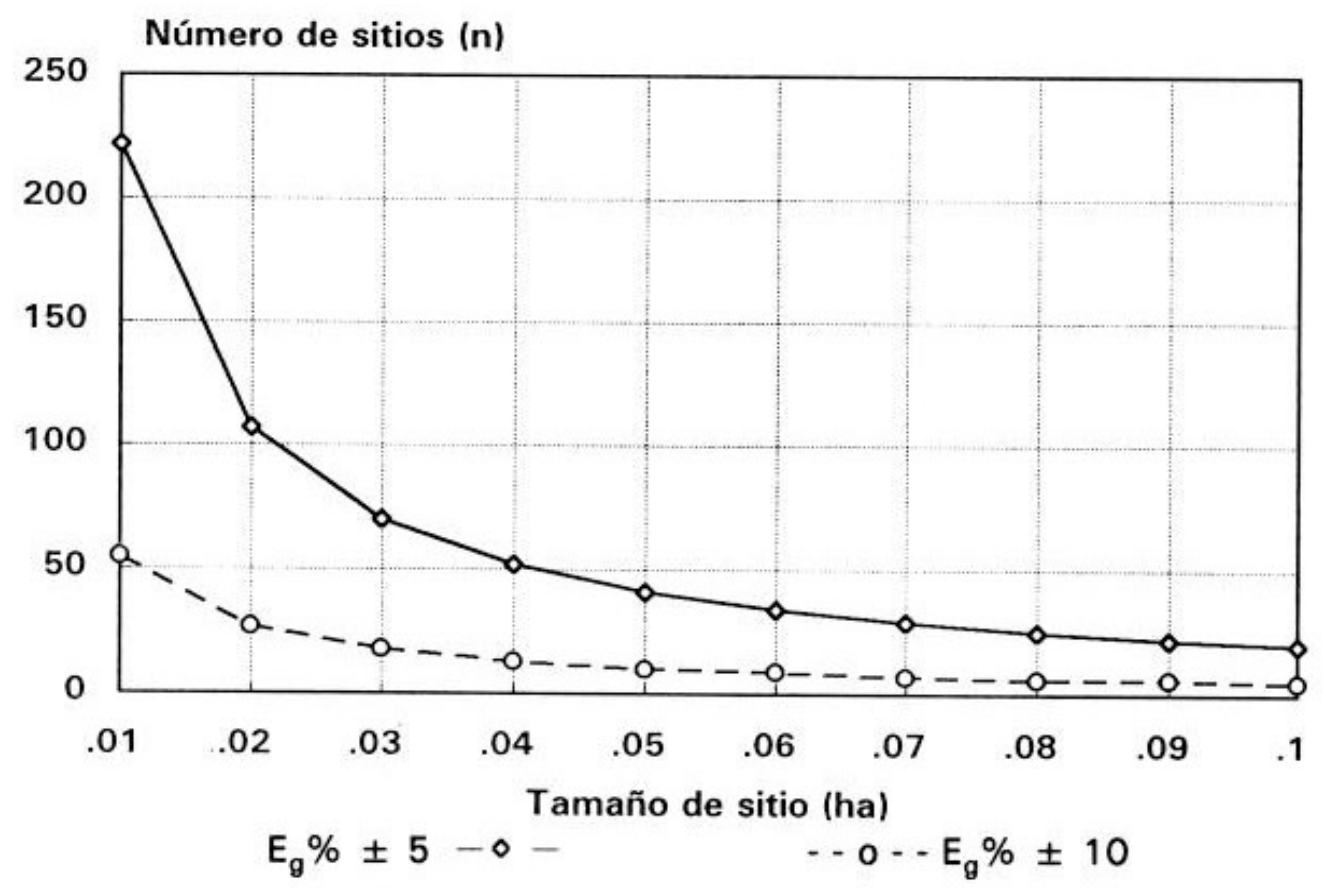

Figura 3. Número de sitios necesario para la estimación del área basal con un error estándar de $\pm 5 \%$ y $\pm 10 \%$ (Minimum plot numbers to estimate basal area with a standard error of $\pm 5 \%$ and $\pm 10 \%$ ) 


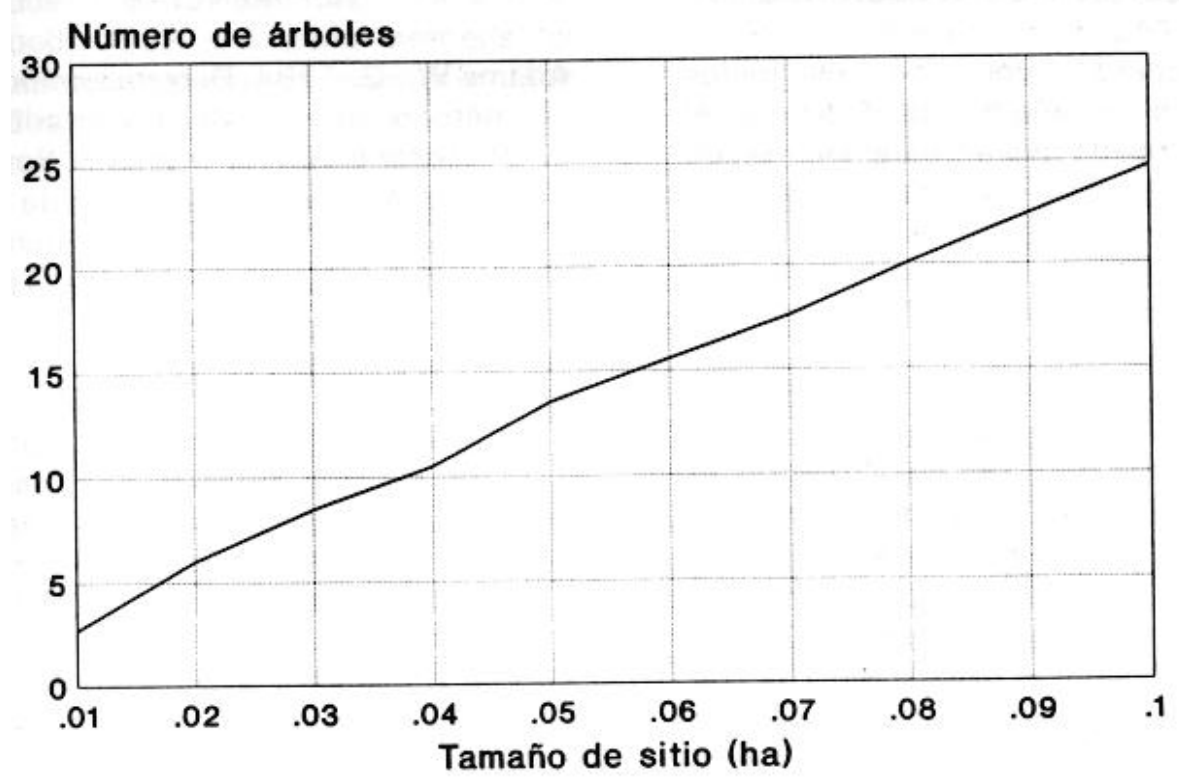

Figura 4. Número de árboles promedio para diferentes tamaños de sitio (Mean tree number for different plot sizes)

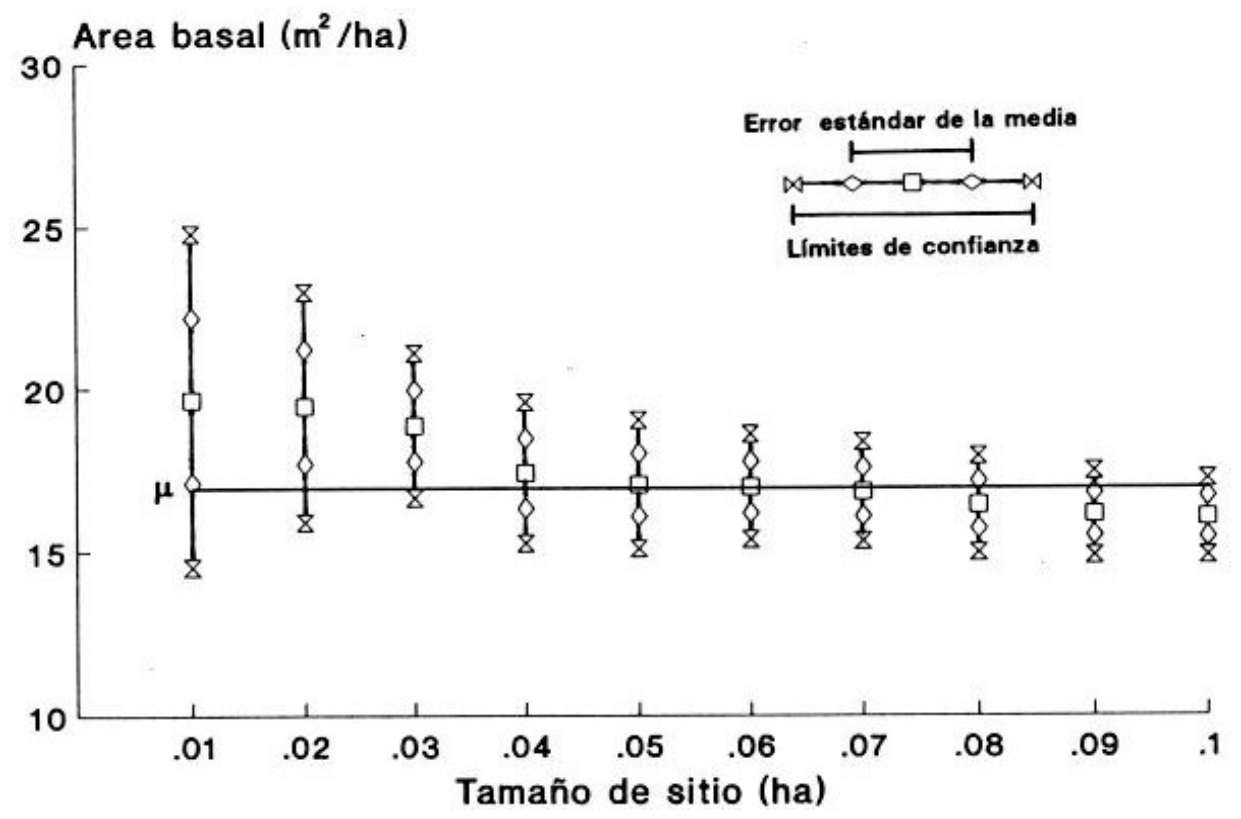

Figura 5. Intervalos del 95\% de confianza del área basal para diferentes tamaños de sitio y área basal media del inventario total $(\mu)$ (Basal area confidence limits for different plot sizes and basal area of the total inventory $(\mu))$ 


\section{DISCUSION Y CONCLUSIONES}

De acuerdo con los resultados obtenidos, el tamaño óptimo de sitio en el rodal investigado puede establecerse en 0.06 ha. Cabe mencionar que en un rodal próximo, una investigación similar (Aguirre et al., 1995) dio como resultado un tamaño de sitio óptimo de 0.04 ha.

La diferencia entre ambos rodales es básicamente la densidad de los mismos (25.66 $\mathrm{m}^{2} / \mathrm{ha}$ de área basal del rodal referido y $16.94 \mathrm{~m}^{2} /$ ha en el investigado en este trabajo). Por otra parte, el resultado obtenido corresponde aproximadamente a los reportados para bosques de coníferas (Avendaño,1991) y de mezquites (Adame,1994); estos estudios determinaron tamaños de sitio óptimo de 0.05 ha. En todos los casos mencionados, los sitios de 0.1 ha empleados convencionalmente resultaron ser muy grandes.

La metodología empleada en este trabajo constituye una alternativa para la racionalización de inventarios forestales en lo que a recursos maderables se refiere. Es necesario, sin embargo, integrar estos procedimientos en un marco general que involucre la evaluación de la condición ecológica de los rodales bajo manejo.

Por otra parte, conviene mencionar que el criterio de optimalidad empleado en este trabajo debe complementarse con investigaciones de los tiempos que involucra la realización de las distintas operaciones que incluye la toma de datos de los inventarios forestales en el terreno.

\section{REFERENCIAS}

A dame W., G. 1994. Determinación de un método óptimo de inventario para Prosopis laevigata (Humb \& Bompl ex Willd) M.C. J honst. en el sur de Nuevo León. Tesis profesional. Facultad de Ciencias Forestales, U.A.N.L. Linares, N.L.

A guirre C., O.A., J . J iménez P. y B. Meraz A. 1995. Optimización de inventarios para manejo forestal: Un caso de estudio en Durango, México. Investigación Agraria: Sistemas y Recursos Forestales 4(1):107-118.

Aguirre C., O.A. y J. J iménez P. 1995. Guía de densidad para Pinus teocote Schl. et Cham. en Nuevo León. Memoria del II Congreso mexicano sobre recursos forestales. Montecillo, Mex. p. 22

Akça, A. y R. Sangen-Emden. 1986. Zur Optimierung von repräsentativen Bestandesaufnahmen. Allgemeine Forst-und J agdzeitung 157(2):43-47

A vendaño A., H.S. 1991. Determinación del tamaño óptimo de sitio de muestreo en un bosque de coníferas en la Sierra de Arteaga, Coah. Tesis profesional. División de Agronomía, U.A.A.A.N. Saltillo, Coah.

De V ries, P.G. 1986. Sampling theory for forest inventory. A teach-yourself course, Springer-Verlag, Berlin.

Kramer, H. y A. Akça. 1987. Leitfaden für Dendrometrie und Bestandesinventur, J .D. Sauerländer's. Verlag.

O'Regan, W. G. y L.G. Arvanitis. 1966. Cost efectiveness in forest sampling. Forest Science 12(4):406-414 
Madera y Bosques 3(1), 1997:71-79

Schreuder H., T., T.G. Gregoire y G.B. Wood. 1993. Sampling methods for multiresource forest inventory. J ohn Wiley \& Sons, Nueva York.
Zeide, B. 1980. Plot size optimization. Forest Science 26 (2):251-257

Zöhrer, F. 1980. Forstinventur. Parey's Studientexte 26. Verlag Paul Parey. Berlin 